George Avalos and Matthew Dvorak (Lincoln, NE)

\title{
A NEW MAXIMALITY ARGUMENT FOR A COUPLED FLUID-STRUCTURE INTERACTION, WITH IMPLICATIONS FOR A DIVERGENCE-FREE FINITE ELEMENT METHOD
}

Abstract. We consider a coupled PDE model of various fluid-structure interactions seen in nature. It has recently been shown by the authors [Contemp. Math. 440, 2007] that this model admits of an explicit semigroup generator representation $\mathcal{A}: D(\mathcal{A}) \subset \mathbf{H} \rightarrow \mathbf{H}$, where $\mathbf{H}$ is the associated space of fluid-structure initial data. However, the argument for the maximality criterion was indirect, and did not provide for an explicit solution $\Phi \in D(\mathcal{A})$ of the equation $(\lambda I-\mathcal{A}) \Phi=F$ for given $F \in \mathbf{H}$ and $\lambda>0$. The present work reconsiders the proof of maximality for the fluid-structure generator $\mathcal{A}$, and gives an explicit method for solving the said fluid-structure equation. This involves a nonstandard usage of the Babuška-Brezzi Theorem. Subsequently, a finite element method for approximating solutions of the fluid-structure dynamics is developed, based upon our explicit proof of maximality.

1. Introduction. In this paper we shall consider a partial differential equation (PDE) system which has been invoked in the existing literature to model various fluid-structure interactions which occur in nature (see, e.g., [13] and [8]). Here, we shall deal strictly with the linearized version of the said fluid-structure PDE model, since (as the reader will see below) the setting of our ideas within a linear framework will be a technical enough topic for one paper. In a follow-up paper, we will consider the Hadamard

2000 Mathematics Subject Classification: 35M10, 65N30.

Key words and phrases: fluid-structure interaction, elimination of pressure, fluidstructure generator, maximal dissipative operator, Ritz-Galerkin method.

The research of George Avalos has been partially supported by NSF Grant DMS0606776 . 
wellposedness of the fully nonlinear fluid-structure PDE, a model which incorporates Navier-Stokes (rather than linear Stokes) flow. Throughout, $\Omega_{\mathrm{f}} \subseteq \mathbb{R}^{n}, n=2$ or 3 , will denote the bounded domain on which the fluid component of the coupled PDE system evolves. The boundary $\partial \Omega_{\mathrm{f}}$ of this domain will be decomposed as $\partial \Omega_{\mathrm{f}}=\Gamma_{\mathrm{s}} \cup \Gamma_{\mathrm{f}}, \Gamma_{\mathrm{s}} \cap \Gamma_{\mathrm{f}}=\emptyset$, with each boundary piece being sufficiently smooth. In addition, $\Omega_{\mathrm{s}}$ will be the domain on which the structural component evolves with time. The coupling between the two distinct fluid and elastic dynamics occurs precisely because $\partial \Omega_{\mathrm{s}}=\Gamma_{\mathrm{s}}$; see Figure 1. That is to say, $\Gamma_{\mathrm{s}}$ will serve as a boundary interface on which certain (to be specified) boundary transmission conditions will exert a strong coupling between the Stokes flow in $\Omega_{\mathrm{f}}$ and the elastic dynamics which are evolving within $\Omega_{\mathrm{s}}$.

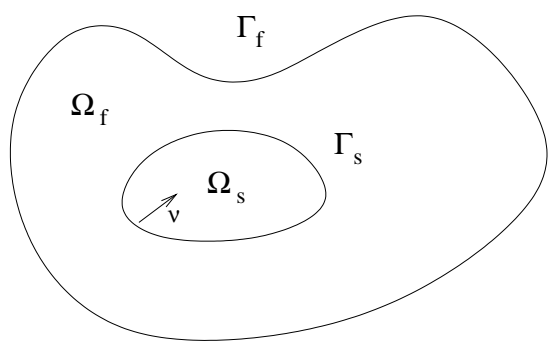

Fig. 1. The fluid-structure geometry

Also, as depicted in Figure 1, $\nu(x)$ will always denote the unit normal vector, which is exterior to $\Omega_{\mathrm{f}}$, and so interior with respect to $\Omega_{\mathrm{s}}$. (This point will be important to bear in mind, as the direction of $\nu$ will influence the computations to be done below.)

With the aforesaid geometrical notions in place, we now proceed to properly introduce the fluid-structure PDE model of our present concern. In dependent variables $u=\left[u_{1}(t, x), \ldots, u_{n}(t, x)\right]$ (the fluid velocity field), $p(t, x)$ (the scalar-valued pressure function), and $w=\left[w_{1}(t, x), \ldots, w_{n}(t, x)\right]$ (the structural displacement field), the following fluid-structure PDE model will be considered:

$$
\begin{aligned}
& (\mathrm{PDE}) \quad \begin{cases}u_{t}-\Delta u+\nabla p=0 & \text { in }(0, T) \times \Omega_{\mathrm{f}}, \\
\operatorname{div}(u)=0 & \text { in }(0, T) \times \Omega_{\mathrm{f}}, \\
w_{t t}-\Delta w+w=0 & \text { in }(0, T) \times \Omega_{\mathrm{s}} ;\end{cases} \\
& (\mathrm{BC}) \quad \begin{cases}\left.u\right|_{\Gamma_{\mathrm{f}}}=0 & \text { on }(0, T) \times \Gamma_{\mathrm{f}}, \\
u=w_{t} & \text { on }(0, T) \times \Gamma_{\mathrm{s}}, \\
\frac{\partial u}{\partial \nu}-\frac{\partial w}{\partial \nu}=p \nu & \text { on }(0, T) \times \Gamma_{\mathrm{s}} ;\end{cases} \\
& (\mathrm{IC}) \quad\left[w(0, \cdot), w_{t}(0, \cdot), u(0, \cdot)\right]=\left[w_{0}, w_{1}, u_{0}\right] \in \mathbf{H},
\end{aligned}
$$


with the space of wellposedness

$$
\mathbf{H} \equiv\left[H^{1}\left(\Omega_{\mathrm{s}}\right)\right]^{n} \times\left[L^{2}\left(\Omega_{\mathrm{s}}\right)\right]^{n} \times \mathcal{H}_{\mathrm{f}},
$$

and with the fluid component space $\mathcal{H}_{\mathrm{f}} \subset\left[L^{2}\left(\Omega_{\mathrm{f}}\right)\right]^{n}$ being defined as follows:

$$
\mathcal{H}_{\mathrm{f}}=\left\{f \in L^{2}\left(\Omega_{\mathrm{f}}\right): \operatorname{div}(f)=0 \text { in } \Omega_{\mathrm{f}} \text { and }[f \cdot \nu]_{\Gamma_{\mathrm{f}}}=0\right\} .
$$

(Recall that if $f \in\left[L^{2}\left(\Omega_{\mathrm{f}}\right)\right]^{n}$ and $\operatorname{div}(f) \in L^{2}\left(\Omega_{\mathrm{f}}\right)$, one has $[f \cdot \nu]_{\partial \Omega_{\mathrm{f}}} \in$ $H^{-1 / 2}\left(\partial \Omega_{\mathrm{f}}\right)$, and so $\mathcal{H}_{\mathrm{f}}$ is well-defined (see $[6$, p. 5$\left.]\right)$. $\mathbf{H}$ is a Hilbert space with the following norm inducing inner product:

$$
\left(\left[\begin{array}{c}
v_{1} \\
v_{2} \\
f
\end{array}\right],\left[\begin{array}{c}
\widetilde{v}_{1} \\
\widetilde{v}_{2} \\
\widetilde{f}
\end{array}\right]\right)_{\mathbf{H}}=\left(\nabla v_{1}, \nabla \widetilde{v}_{1}\right) \Omega_{\mathrm{s}}+\left(v_{1}, \widetilde{v}_{1}\right) \Omega_{\mathrm{s}}+\left(v_{2}, \widetilde{v}_{2}\right) \Omega_{\mathrm{s}}+(f, \widetilde{f})_{\Omega_{\mathrm{f}}}
$$

(here of course, $(f, g)_{\Omega} \equiv \int_{\Omega} f g d \Omega$ ).

Our objective here will be to ascertain wellposedness of the PDE model (1.1)-(1.3) for initial data $\left[w_{0}, w_{1}, u_{0}\right]$ in the apparently "natural" space $\mathbf{H}$ of finite energy. Here at the start, we should emphasize that even for this purely linear problem, wellposedness of the dynamics is far from a pedestrian exercise. In fact, the primary difficulty, and one which will drive our methodology below, lies in an appropriate elimination of the pressure term $p(t, x)$. In classical Navier-Stokes theory, which involves uncoupled fluid flow, such pressure elimination is accomplished by means of the famed Leray (or Helmholtz) projector (see [6]). However, a legitimate application of the Leray projector is based upon a presupposition that the fluid velocity field satisfies the so-called "no slip" boundary condition, i.e., $\left.u\right|_{\partial \Omega_{\mathrm{f}}}=0$. In our present situation, the no slip boundary conditions will certainly not be in play, inasmuch as the fluid component of (1.1)-(1.3) is coupled to the structural component via the boundary interface $\Gamma_{\mathrm{s}}$. Thus, the use of the Leray projector is wholly invalid here; consequently, the pressure term in our fluid-structure interaction must be eliminated by nonstandard means.

In this connection, we should note the work in [4], which deals with linear and nonlinear versions of the fluid-structure system (1.1)-(1.3). In [4], an elimination of the pressure, which again is a sine qua non for the resolution of the fluid-structure dynamics, is obtained by equating (1.1)-(1.3) with an appropriate variational relation. Thus in [4], issues of wellposedness (and of regularity) for (1.1)-(1.3) are subsequently considered within the context of an associated variational relation, or weak form.

On the other hand, in [1], the pressure is eliminated by the very different means of identifying the pressure term $p(t, x)$ in (1.1)-(1.3) as the solution of a certain elliptic boundary value problem (BVP). This BVP contains forcing interior and boundary terms comprised of the fluid and structure variables $u$ and $w$, as well as (boundary traces) of their derivatives. By writing out 
$p(t, x)$ as the solution of the said BVP (through relevant Green's operators), the authors in [1] are able to give an explicit semigroup generator representation for the fluid-structure dynamics (1.1)-(1.3). (And of course, having in hand such a fluid-structure generator, one can, in principle, attempt to glean useful qualitative information for solutions of (1.1)-(1.3); e.g., spectral results for the generator, stability of the semigroup, observability inequalities which are dual to certain boundary controllability problems, and so on.)

A portion of the present paper is essentially a revisiting of the wellposedness work in [1]: As we noted, the nonstandard elimination of the pressure term $p$ in [1], by its association with an appropriate elliptic BVP, eventually allows for an explicit semigroup generator formulation of the fluid-structure model (1.1)-(1.3). In fact, the generator $\mathcal{A}: D(\mathcal{A}) \subset \mathbf{H} \rightarrow \mathbf{H}$ is given explicitly in (3.2) below.

It is justified in [1] that the fluid-structure generator $\mathcal{A}: D(\mathcal{A}) \subset \mathbf{H} \rightarrow \mathbf{H}$ is maximal dissipative, and so by an ostensibly classical invocation of the Lumer-Phillips theorem, the fluid-structure model $\mathcal{A}$ generates a contraction semigroup on $\mathbf{H}$; however, the proof of maximality in [1] is given by an indirect argument. Namely, maximality is inferred by deriving a necessary upper bound for the resolvent operator $\mathcal{R}(\lambda ; \mathcal{A})$, where $\lambda>0$, from which the range condition Range $(\lambda I-\mathcal{A})=\mathbf{H}$ can subsequently be deduced by classical functional analysis; see, e.g., Theorem 1.2 of [16].

Thus, although the work in [1] justifies the maximality condition Range $(\lambda I-\mathcal{A})=\mathbf{H}$ for $\lambda>0$, the following is not explicitly shown: Given arbitrary fluid-structure data $\left[v_{1}^{*}, v_{2}^{*}, f^{*}\right] \in \mathbf{H}$, how can one find an element $\left[v_{1}, v_{2}, f\right] \in D(\mathcal{A})$ (to be specified below) which solves the static fluid-structure PDE,

$$
(\lambda I-\mathcal{A})\left[\begin{array}{c}
v_{1} \\
v_{2} \\
f
\end{array}\right]=\left[\begin{array}{l}
v_{1}^{*} \\
v_{2}^{*} \\
f^{*}
\end{array}\right] ?
$$

(note that from the underlying dissipativity of the operator $\mathcal{A}: D(\mathcal{A}) \subset$ $\mathbf{H} \rightarrow \mathbf{H}$, as given below in (3.2), the solution $\left[v_{1}, v_{2}, f\right]$ to the equation above will be unique).

Accordingly, the present paper is partially devoted to giving an explicit methodology for solving the abstract equation (1.4). This work will involve a nonstandard usage of the Babuška-Brezzi Theorem, as we shall see below.

As an immediate implication of our novel maximality argument, one can subsequently devise a finite element method (FEM) by which to approximate solutions to the fluid-structure PDE model (1.1)-(1.3). This will be illustrated at the tail end of our paper, within the context of a particular static example. (And of course having in hand a systematic way to 
solve (1.4), one can subsequently proceed to solve the time evolving system (1.1)-(1.3), for Cauchy data $\left[w_{0}, w_{1}, u_{0}\right] \in \mathbf{H}$, by invoking the exponential formula for $\left\{e^{\mathcal{A} t}\right\}_{t \geq 0}$, and corresponding implicit schemes; see, e.g., [15].) We should also emphasize here that due to our nonstandard invocation of Babuška-Brezzi in the course of solving (1.4), the fluid basis functions, adopted in the FEM for numerically approximating the solution of (1.4), will not need to be divergence free.

2. Elimination of the pressure. As we have said, a successful resolution of the fluid-structure system (1.1)-(1.3) depends upon an appropriate elimination of the pressure, which by circumstance cannot involve the Leray projector. To this end, we will maneuver as in the paper [1], so as to identify the pressure term $p(t, \cdot)$ as the solution of a certain elliptic BVP. (We might also note that, at least formally, the association of pressure functions with elliptic BVP's, at least in the context of uncoupled fluid flow problems, has been long known, but generally not exploited; see, e.g., [7].)

Because of the overarching importance of these steps to eliminate the pressure, we will include them here, rather than merely appeal directly to [1]; else the reader would have absolutely no insight as to why the fluidstructure generator $\mathcal{A}: D(\mathcal{A}) \subset \mathbf{H} \rightarrow \mathbf{H}$ assumes the appearance it does in (3.2).

The elimination of the pressure is based upon the following observation: For fixed $t \in(0, T)$, the scalar-valued function $p(t, x)$ solves the following elliptic BVP:

$$
\begin{aligned}
\Delta p & =0 & & \text { in } \Omega_{\mathrm{f}}, \\
p & =\frac{\partial u}{\partial \nu} \cdot \nu-\frac{\partial w}{\partial \nu} \cdot \nu & & \text { on } \Gamma_{\mathrm{s}}, \\
\frac{\partial p}{\partial \nu} & =(\Delta u) \cdot \nu & & \text { on } \Gamma_{\mathrm{f}} .
\end{aligned}
$$

This BVP is derived, pointwise in time, in the following way: (i) Taking the divergence of both sides of the fluid PDE in (1.1), and using $\operatorname{div}(u)=0$, one has (2.1). (ii) Moreover, the expression (2.2) on the boundary interface $\Gamma_{\mathrm{s}}$ is attained by taking the dot product of both sides of the Neumann boundary condition in (1.2), with respect to the unit normal vector $\nu(x)$. (iii) Finally, the boundary condition (2.3) is obtained by taking the dot product of both sides of the fluid PDE with respect to an appropriate extension of the normal vector $\nu(x)$, and restricting the resulting quantity to $\Gamma_{\mathrm{f}}$ (implicitly using the fact that $\left.[u \cdot \nu]_{\Gamma_{\mathrm{f}}}=0\right)$. We now proceed to "solve" this system (2.1)-(2.3), by means of abstract Green's maps which account for the contribution of boundary data (see, e.g., the many references in [12], wherein this ostensibly classical idea first germinated in PDE control theory): Let the respective 
Dirichlet and Neumann maps $D_{\mathrm{s}}: L^{2}\left(\Gamma_{\mathrm{s}}\right) \rightarrow L^{2}\left(\Omega_{\mathrm{f}}\right), N_{\mathrm{f}}: L^{2}\left(\Gamma_{\mathrm{f}}\right) \rightarrow L^{2}\left(\Omega_{\mathrm{f}}\right)$ be given by:

$$
\begin{array}{r}
h=D_{\mathrm{s}}(g) \Leftrightarrow \begin{cases}\Delta h=0 & \text { in } \Omega_{\mathrm{f}}, \\
h=g & \text { on } \Gamma_{\mathrm{s}}, \\
\frac{\partial h}{\partial \nu}=0 & \text { on } \Gamma_{\mathrm{f}},\end{cases} \\
h=N_{\mathrm{f}}(g) \Leftrightarrow \begin{cases}\Delta h=0 & \text { in } \Omega_{\mathrm{f}}, \\
h=0 & \text { on } \Gamma_{\mathrm{s}}, \\
\frac{\partial h}{\partial \nu}=g & \text { on } \Gamma_{\mathrm{f}},\end{cases}
\end{array}
$$

(and so each map gives rise to a harmonic extension of boundary data). Then by elliptic regularity (see, e.g., [14]), the maps satisfy, for all real $r$,

$$
D_{\mathrm{s}} \in \mathcal{L}\left(H^{r}\left(\Gamma_{\mathrm{s}}\right), H^{r+1 / 2}\left(\Omega_{\mathrm{f}}\right)\right), \quad N_{\mathrm{f}} \in \mathcal{L}\left(H^{r}\left(\Gamma_{\mathrm{f}}\right), H^{r+3 / 2}\left(\Omega_{\mathrm{f}}\right)\right) .
$$

With this operator-theoretic machinery in hand, the solution of $(2.1)-(2.3)$ can then be written, for fixed $t \in(0, T)$, as

$$
p(t)=D_{\mathrm{s}}\left[\left(\frac{\partial u(t)}{\partial \nu} \cdot \nu-\frac{\partial w(t)}{\partial \nu} \cdot \nu\right)_{\Gamma_{\mathrm{s}}}\right]+N_{\mathrm{f}}\left[(\Delta u(t) \cdot \nu)_{\Gamma_{\mathrm{f}}}\right] \quad \text { in } \Omega_{\mathrm{f}} .
$$

If we now define the linear maps $G_{1}$ and $G_{2}$ via

$$
\begin{aligned}
G_{1} w & \equiv \nabla\left(D_{\mathrm{s}}\left[\left(\frac{\partial w}{\partial \nu} \cdot \nu\right)_{\Gamma_{\mathrm{s}}}\right]\right), \\
G_{2} u & \equiv-\nabla\left(D_{\mathrm{s}}\left[\left(\frac{\partial u}{\partial \nu} \cdot \nu\right)_{\Gamma_{\mathrm{s}}}\right]+N_{\mathrm{f}}\left[(\Delta u \cdot \nu)_{\Gamma_{\mathrm{f}}}\right]\right),
\end{aligned}
$$

then these and the expression in (2.6) allow one to write the fluid PDE component of the system (1.1)-(1.3) in terms of $u$ and $w$ alone; that is, pressure is eliminated from the Stokes equation in (1.1)-(1.3), so as to have

$$
u_{t}=\Delta u+G_{1} w+G_{2} u \quad \text { in }(0, T) \times \Omega_{\mathrm{f}} .
$$

\section{The fluid-structure generator and its domain of definition}

3.1. The explicit form of the generator. Owing to the maps $G_{i}$, defined in (2.7) and (2.8), which allow the fluid flow component of (1.1)-(1.3) to be rewritten as the pressure-free equation (2.9), we can now construct a linear operator $\mathcal{A}: D(\mathcal{A}) \subset \mathbf{H} \rightarrow \mathbf{H}$ which can be used to abstractly model (1.1)-(1.3): To wit, the fluid-structure system (1.1)-(1.3) may be written as

$$
\begin{aligned}
\frac{d}{d t}\left[\begin{array}{c}
w \\
w_{t} \\
u
\end{array}\right] & =\mathcal{A}\left[\begin{array}{c}
w \\
w_{t} \\
u
\end{array}\right], \\
{\left[w(0), w_{t}(0), u(0)\right] } & =\left[w_{0}, w_{1}, u_{0}\right] \in \mathbf{H},
\end{aligned}
$$


where

$$
\mathcal{A} \equiv\left[\begin{array}{ccc}
0 & I & 0 \\
\Delta-I & 0 & 0 \\
G_{1} & 0 & \Delta+G_{2}
\end{array}\right]
$$

Of course, the domain of definition, $D(\mathcal{A}) \subset \mathbf{H} \rightarrow \mathbf{H}$, must also be specified here. In addition, and much more to the point: If $\mathcal{A}: D(\mathcal{A}) \subset \mathbf{H} \rightarrow \mathbf{H}$ is to generate a semigroup $\left\{e^{\mathcal{A} t}\right\}_{t \geq 0} \subset \mathcal{L}(\mathbf{H})$ - and so the solution $\left[w(t), w_{t}(t), u(t)\right]$ to (1.1)-(1.3) is obtained by applying $e^{\mathcal{A} t}$ to initial data $\left[w_{0}, w_{1}, u_{0}\right]$ - then the domain $D(\mathcal{A})$ should be constructed so as to allow for the existence of just such a semigroup. In particular, if $\mathcal{A}: \mathbf{H} \rightarrow \mathbf{H}$ is maximal dissipative with respect to the specified domain $D(\mathcal{A})$, then $\left\{e^{\mathcal{A} t}\right\}_{t \geq 0} \subset \mathcal{L}(\mathbf{H})$ would exist as a contraction $C_{0}$ semigroup, by the Lumer-Phillips theorem.

Therefore, an appropriate definition of $D(\mathcal{A})$ is all important here. Our statement concerning the $D(\mathcal{A})$ will be explicit and outright. This is in contrast to what was done in $[1]$, wherein $\operatorname{Range}(\mathcal{A})$ is first carefully characterized $\left(^{1}\right)$, and then the domain $D(\mathcal{A})$, with all its intrinsic features, is given via the relation $D(\mathcal{A})=\mathcal{A}^{-1}(\operatorname{Range}(\mathcal{A}))$; see Theorem 2.1 of $[1]$. (We should also make mention of the paper [2], in which a generator representation is derived for a more complicated fluid-structure system, involving Stokes flow and the Lamé system of elasticity, with the generator domain also being explicitly identified.)

Before stating $D(\mathcal{A})$ explicitly, we first need some preliminaries.

Proposition 1. Suppose an $L^{2}\left(\Omega_{\mathrm{f}}\right)$-function $\varrho$ satisfies $\Delta \varrho \in L^{2}\left(\Omega_{\mathrm{f}}\right)$. Then one has the following boundary trace estimate:

$$
\begin{aligned}
\left\|\left.\varrho\right|_{\partial \Omega_{\mathrm{f}}}\right\|_{H^{-1 / 2}\left(\partial \Omega_{\mathrm{f}}\right)}+\left\|\left.\frac{\partial \varrho}{\partial \nu}\right|_{\partial \Omega_{\mathrm{f}}}\right\|_{H^{-3 / 2}\left(\partial \Omega_{\mathrm{f}}\right)} & \leq C\left\{\|\varrho\|_{L^{2}\left(\Omega_{\mathrm{f}}\right)}+\|\Delta \varrho\|_{L^{2}\left(\Omega_{\mathrm{f}}\right)}\right\} .
\end{aligned}
$$

Proof. Since the Sobolev trace map

$$
\gamma \in \mathcal{L}\left(H^{2}\left(\Omega_{\mathrm{f}}\right), H^{3 / 2}\left(\partial \Omega_{\mathrm{f}}\right) \times H^{1 / 2}\left(\partial \Omega_{\mathrm{f}}\right)\right)
$$

is surjective, where $\gamma f=\left[\left.f\right|_{\partial \Omega_{\mathrm{f}}},\left.\frac{\partial f}{\partial \nu}\right|_{\partial \Omega_{\mathrm{f}}}\right]$ for $f \in C^{\infty}\left(\bar{\Omega}_{\mathrm{f}}\right)$, there exists a continuous right inverse $\gamma^{+} \in \mathcal{L}\left(H^{3 / 2}\left(\partial \Omega_{\mathrm{f}}\right) \times H^{1 / 2}\left(\partial \Omega_{\mathrm{f}}\right), H^{2}\left(\Omega_{\mathrm{f}}\right)\right)$. That is, $\gamma \gamma^{+}\left(\left[\phi_{1}, \phi_{2}\right]\right)=\left[\phi_{1}, \phi_{2}\right]$ for all $\left[\phi_{1}, \phi_{2}\right] \in H^{3 / 2}\left(\partial \Omega_{\mathrm{f}}\right) \times H^{1 / 2}\left(\partial \Omega_{\mathrm{f}}\right)$. Thus, by Green's Theorem we have for $\varrho$ (initially smooth enough) and any $\left[\phi_{1}, \phi_{2}\right] \in$ $H^{3 / 2}\left(\partial \Omega_{\mathrm{f}}\right) \times H^{1 / 2}\left(\partial \Omega_{\mathrm{f}}\right)$,

$\left({ }^{1}\right)$ In fact, it is shown in [1] that $\operatorname{Range}(\mathcal{A})=\left\{\left[g_{0}, g_{1}, f_{0}\right] \in \mathbf{H}: \int_{\Gamma_{\mathrm{s}}} g_{0} \cdot \nu d \Gamma_{\mathrm{s}}=0\right\}$. 


$$
\begin{aligned}
\int_{\partial \Omega_{\mathrm{f}}} \varrho \phi_{2} d \partial \Omega_{\mathrm{f}} \\
\quad=\int_{\Omega_{\mathrm{f}}} \varrho \Delta \gamma^{+}\left(\left[\phi_{1}, \phi_{2}\right]\right) d \Omega_{\mathrm{f}}+\int_{\Omega_{\mathrm{s}}} \nabla \varrho \cdot \nabla \gamma^{+}\left(\left[\phi_{1}, \phi_{2}\right]\right) d \Omega_{\mathrm{f}} \\
=\int_{\Omega_{\mathrm{f}}} \varrho \Delta \gamma^{+}\left(\left[\phi_{1}, \phi_{2}\right]\right) d \Omega_{\mathrm{f}}-\int_{\Omega_{\mathrm{f}}} \Delta \varrho \gamma^{+}\left(\left[\phi_{1}, \phi_{2}\right]\right) d \Omega_{\mathrm{f}}+\int_{\partial \Omega_{\mathrm{f}}} \frac{\partial \varrho}{\partial \nu} \phi_{1} d \partial \Omega_{\mathrm{f}},
\end{aligned}
$$

whence we obtain the estimate (3.3), for smooth $\varrho$. A density argument concludes the proof.

Lemma 2. Suppose a pair $(\mu, \varrho) \in\left[L^{2}\left(\Omega_{\mathrm{f}}\right)\right]^{n} \times L^{2}\left(\Omega_{\mathrm{f}}\right)$ has the following properties:

(i) $\mu \in\left[H^{1}\left(\Omega_{\mathrm{f}}\right)\right]^{n}$;

(ii) $\operatorname{div}(\mu)=0$;

(iii) $-\Delta \mu+\nabla \varrho=h$, where $h \in\left[L^{2}\left(\Omega_{\mathrm{f}}\right)\right]^{n}$ and $\operatorname{div}(h)=0$.

Then one has the additional boundary regularity for the pair $(\mu, \varrho)$ :

$$
\begin{array}{cr}
\left.\varrho\right|_{\partial \Omega_{\mathrm{f}}} \in H^{-1 / 2}\left(\partial \Omega_{\mathrm{f}}\right), & \left.\frac{\partial \varrho}{\partial \nu}\right|_{\partial \Omega_{\mathrm{f}}} \in H^{-3 / 2}\left(\partial \Omega_{\mathrm{f}}\right), \\
\left.\frac{\partial \mu}{\partial \nu}\right|_{\partial \Omega_{\mathrm{f}}} \in\left[H^{-1 / 2}\left(\partial \Omega_{\mathrm{f}}\right)\right]^{n}, & {[(\Delta \mu) \cdot \nu]_{\partial \Omega_{\mathrm{f}}} \in H^{-3 / 2}\left(\partial \Omega_{\mathrm{f}}\right) .}
\end{array}
$$

Proof. From the assumption in (iii) we infer that the $L^{2}$-function $\varrho$ is harmonic: in fact, taking the divergence of both sides of the equation in (iii), we have

$$
\Delta \varrho=\operatorname{div}(\Delta \mu)+\operatorname{div}(h)=0,
$$

since $\mu$ and the forcing term $h$ are each solenoidal. Consequently, Proposition 1 provides a meaning (continuously) to the boundary traces

$$
\left.\varrho\right|_{\partial \Omega_{\mathrm{f}}} \in H^{-1 / 2}\left(\partial \Omega_{\mathrm{f}}\right),\left.\quad \frac{\partial \varrho}{\partial \nu}\right|_{\partial \Omega_{\mathrm{f}}} \in H^{-3 / 2}\left(\partial \Omega_{\mathrm{f}}\right) .
$$

In turn, the given Dirichlet trace for square integrable $\varrho$, combined with an application of Green's formula, shows that $\nabla \varrho$ is in $\left[\left(H^{1}\left(\Omega_{\mathrm{f}}\right)\right)^{n}\right]^{\prime}$, not merely in $\left(H^{-1}\left(\Omega_{\mathrm{f}}\right)\right)^{n}$. Therefore, the variable $\mu \in\left[H^{1}\left(\Omega_{\mathrm{f}}\right)\right]^{n}$ satisfies the elliptic equation

$$
-\Delta \mu=-\nabla \varrho+h \in\left[\left(H^{1}\left(\Omega_{\mathrm{f}}\right)\right)^{n}\right]^{\prime} .
$$

Appealing then to elliptic theory (see, e.g., p. 71, Theorem 3.8.1 of [10]), we see that continuously,

$$
\left.\frac{\partial \mu}{\partial \nu}\right|_{\partial \Omega_{\mathrm{f}}} \in\left[H^{-1 / 2}\left(\partial \Omega_{\mathrm{f}}\right)\right]^{n}
$$


Finally, taking the dot product of both sides of the equation in (iii) with an appropriate extension of the normal vector, and restricting the resulting relation to $\partial \Omega_{\mathrm{f}}$, we obtain

$$
[(\Delta \mu) \cdot \nu]_{\partial \Omega_{\mathrm{f}}}=\left.\frac{\partial \varrho}{\partial \nu}\right|_{\partial \Omega_{\mathrm{f}}}-[h \cdot \nu]_{\partial \Omega_{\mathrm{f}}} \in H^{-3 / 2}\left(\Omega_{\mathrm{f}}\right)
$$

(implicitly, we are also using the fact that since $h \in\left[L^{2}\left(\Omega_{\mathrm{f}}\right)\right]^{3}$ and $\operatorname{div}(h)=0$, the trace $[h \cdot \nu]_{\partial \Omega_{\mathrm{f}}}$ is well-defined as an element in $H^{-1 / 2}\left(\partial \Omega_{\mathrm{f}}\right)$; see Proposition 1.4 of [6]). The procurement of (3.4), (3.5) and (3.6) now completes the proof of Lemma 2.

3.2. Domain of $\mathcal{A}: \mathbf{H} \rightarrow \mathbf{H}$. We are now in a position to set the domain of the operator $\mathcal{A}: \mathbf{H} \rightarrow \mathbf{H}$, as defined in (3.2). The subspace $D(\mathcal{A})$ is composed of all $\left[w_{0}, w_{1}, u_{0}\right] \in \mathbf{H}$ which satisfy the following:

(A.1) $\left[w_{0}, w_{1}, u_{0}\right] \in\left[H^{1}\left(\Omega_{\mathrm{s}}\right)\right]^{n} \times\left[H^{1}\left(\Omega_{\mathrm{s}}\right)\right]^{n} \times\left(\left[H^{1}\left(\Omega_{\mathrm{f}}\right)\right]^{n} \cap \mathcal{H}_{\mathrm{f}}\right.$.

(A.2) On the boundary portion $\Gamma_{\mathrm{f}}$, the fluid component $\left.u_{0}\right|_{\Gamma_{\mathrm{f}}}$ is zero.

(A.3) The structural component $w_{0}$ satisfies $\Delta w_{0} \in\left[L^{2}\left(\Omega_{\mathrm{s}}\right)\right]^{n}$. (So by elliptic theory $\left.\frac{\partial w_{0}}{\partial \nu}\right|_{\Gamma_{\mathrm{s}}}$ is well-defined as an element of $\left[H^{-1 / 2}\left(\Gamma_{\mathrm{S}}\right)\right]^{n}$; see, e.g., p. 71, Theorem 3.8.1 of [10].)

(A.4) The components obey the following relation on the boundary interface $\Gamma_{\mathrm{s}}$ :

$$
u_{0}=w_{1} \quad \text { on } \Gamma_{\mathrm{s}} .
$$

(A.5) For the given data $\left[w_{0}, w_{1}, u_{0}\right]$, there exists a corresponding "pressure" function $\pi_{0} \in L^{2}\left(\Omega_{\mathrm{f}}\right)$ such that:

(A.5a) The pair $\left(u_{0}, \pi_{0}\right)$ satisfies

$$
-\Delta u_{0}+\nabla \pi_{0} \in \mathcal{H}_{\mathrm{f}} .
$$

Consequently, we have, continuously, by Lemma 2,

$$
\begin{gathered}
\left.\pi_{0}\right|_{\partial \Omega_{\mathrm{f}}} \in H^{-1 / 2}\left(\partial \Omega_{\mathrm{f}}\right),\left.\quad \frac{\partial \pi_{0}}{\partial \nu}\right|_{\partial \Omega_{\mathrm{f}}} \in H^{-3 / 2}\left(\partial \Omega_{\mathrm{f}}\right), \\
\left.\frac{\partial u_{0}}{\partial \nu}\right|_{\partial \Omega_{\mathrm{f}}} \in\left[H^{-1 / 2}\left(\partial \Omega_{\mathrm{f}}\right)\right]^{n}, \quad\left[\left(\Delta u_{0}\right) \cdot \nu\right]_{\partial \Omega_{\mathrm{f}}} \in H^{-3 / 2}\left(\partial \Omega_{\mathrm{f}}\right) .
\end{gathered}
$$

(A.5b) The components $\left[u_{0}, w_{0}\right]$ and the associated pressure function $\pi_{0}$ obey the following relation on the boundary interface $\Gamma_{\mathrm{s}}$ :

$$
\frac{\partial u_{0}}{\partial \nu}-\frac{\partial w_{0}}{\partial \nu}=\pi_{0} \nu \quad \text { on } \Gamma_{\mathrm{s}}
$$


Note that, as we showed outright in generating the BVP (2.1)-(2.3), the function $\pi_{0}$ a fortiori satisfies the following BVP:

$$
\begin{aligned}
\Delta \pi_{0} & =0 \text { in } \Omega_{\mathrm{f}}, \\
\frac{\partial \pi_{0}}{\partial \nu} & =(\Delta u) \cdot \nu \quad \text { on } \Gamma_{\mathrm{f}}, \\
\pi_{0} & =\left(\frac{\partial u_{0}}{\partial \nu}-\frac{\partial w_{0}}{\partial \nu}\right) \cdot \nu \quad \text { on } \Gamma_{\mathrm{s}} .
\end{aligned}
$$

Consequently, we can then use the elliptic maps $D_{\mathrm{s}}$ and $N_{\mathrm{f}}$, defined respectively in (2.4) and (2.5), so as to identify this pressure $\pi_{0}$ associated with the triple $\left[w_{0}, w_{1}, u_{0}\right]$, through the relation

$$
\begin{aligned}
\pi_{0} & =p\left(u_{0}, w_{0}\right) \\
& =D_{\mathrm{s}}\left\{\left[\left(\frac{\partial u_{0}}{\partial \nu}-\frac{\partial w_{0}}{\partial \nu}\right) \cdot \nu\right]_{\Gamma_{\mathrm{s}}}\right\}+N_{\mathrm{f}}\left\{\left[\left(\Delta u_{0}\right) \cdot \nu\right]_{\Gamma_{\mathrm{f}}}\right\},
\end{aligned}
$$

whence we obtain

$$
\nabla \pi_{0}=\nabla p\left(u_{0}, w_{0}\right)=-G_{1} w_{0}-G_{2} u_{0}
$$

where the $G_{i}$ are as defined in (2.7) and (2.8). Note how the trace regularity result in Lemma 2 has allowed for a meaningful application of the maps $G_{1}$ and $G_{2}$. This means that our definition $(\mathrm{A} 1)-(\mathrm{A} 5)$ of $D(\mathcal{A})$ is consistent in that one truly has

$$
\left[\begin{array}{l}
w_{0} \\
w_{1} \\
u_{0}
\end{array}\right] \in D(\mathcal{A}) \Rightarrow \mathcal{A}\left[\begin{array}{l}
w_{0} \\
w_{1} \\
u_{0}
\end{array}\right] \in \mathbf{H} .
$$

3.3. Semigroup wellposedness of (1.1)-(1.3). For the fluid structure mo$\operatorname{del} \mathcal{A}: D(\mathcal{A}) \subset \mathbf{H} \rightarrow \mathbf{H}$, as defined in (3.2) and (A1)-(A5), we do in fact have semigroup generation.

Theorem 3.

(i) The operator $\mathcal{A}: D(\mathcal{A}) \subset \mathbf{H} \rightarrow \mathbf{H}$ generates a contraction $C_{0}$ semigroup $\left\{e^{\mathcal{A} t}\right\}_{t \geq 0}$ on $\mathbf{H}$. Thus, given $\left[w_{0}, w_{1}, u_{0}\right] \in \mathbf{H}$, the solution $\left[w, w_{t}, u\right] \in C([0, T] ; \mathbf{H})$ of (1.1)-(1.3) is given by

$$
\left[\begin{array}{c}
w(t) \\
w_{t}(t) \\
u(t)
\end{array}\right]=e^{\mathcal{A} t}\left[\begin{array}{l}
w_{0} \\
w_{1} \\
u_{0}
\end{array}\right] .
$$

Moreover, the fluid component has the additional regularity

$$
u \in L^{2}\left(0, T ;\left[H^{1}\left(\Omega_{\mathrm{f}}\right)\right]^{n}\right) .
$$

(ii) If $\left[w_{0}, w_{1}, u_{0}\right] \in D(\mathcal{A})$, then one has the additional regularity for the solution $\left[w, w_{t}, u\right]$ of $(1.1)-(1.3)$ : 
(ii.a) $\left[w, w_{t}, u\right] \in C([0, T] ; D(\mathcal{A})), p \in C\left([0, T] ; L^{2}\left(\Omega_{\mathrm{f}}\right)\right)$, with pressure $p$ being given by the expression

(ii.b) $p=D_{\mathrm{s}}\left\{\left[\left(\frac{\partial u}{\partial \nu}-\frac{\partial w}{\partial \nu}\right) \cdot \nu\right]_{\Gamma_{\mathrm{s}}}\right\}+N_{\mathrm{f}}\left\{[(\Delta u) \cdot \nu]_{\Gamma_{\mathrm{f}}}\right\}$.

Combining the regularity for $u$ in (3.8) with the Sobolev Trace Theorem and the boundary condition in (1.2), we also have

Corollary 4. Given $\left[w_{0}, w_{1}, u_{0}\right] \in \mathbf{H}$, the mechanical velocity component of the solution $\left[w, w_{t}, u\right]$ to $(1.1)-(1.3)$ satisfies

$$
\left.w_{t}\right|_{\Gamma_{\mathrm{s}}} \in L^{2}\left(0, T ;\left[H^{1 / 2}\left(\Gamma_{\mathrm{s}}\right)\right]^{n}\right) .
$$

The proof of Theorem 3(i) is given in the next section. On establishing the existence of a fluid-structure semigroup $\left\{e^{\mathcal{A} t}\right\}_{t \geq 0} \subset \mathcal{L}(\mathbf{H})$, Theorem 3(ii) is an immediate consequence of semigroup theory (see, e.g., [15]) and the necessary expression for the pressure function given in (3.7).

As we said in our earlier remarks, Theorem 3 was originally proved in [1]. We will give here, however, a very different maximality argument than that presented in [1], an argument which will ultimately allow for a divergence free FEM method.

\section{The proof of Theorem 3}

4.1. The proof of the dissipativity of $\mathcal{A}: D(\mathcal{A}) \subset \mathbf{H} \rightarrow \mathbf{H}$. The dissipativity argument has been provided in [1], but we sketch it here for the sake of completeness, at least for real-valued functions. If $\left[v_{1}, v_{2}, f\right] \in D(\mathcal{A})$, then there exists a pressure function $\pi \in L^{2}\left(\Omega_{\mathrm{f}}\right)$ such that $\left[v_{1}, v_{2}, f, \pi\right]$ has the properties (A1)-(A5) above. Thus, all the computations to be done below are wholly justified: Through the definition of $\mathcal{A}: D(\mathcal{A}) \subset \mathbf{H} \rightarrow \mathbf{H}$ in (3.2), we have

$$
\begin{aligned}
& \left(\mathcal{A}\left[\begin{array}{c}
v_{1} \\
v_{2} \\
f
\end{array}\right],\left[\begin{array}{c}
v_{1} \\
v_{2} \\
f
\end{array}\right]\right)_{\mathbf{H}}=\left(\left[\begin{array}{c}
v_{2} \\
\Delta v_{1}-v_{1} \\
\Delta f-\nabla \pi
\end{array}\right],\left[\begin{array}{c}
v_{1} \\
v_{2} \\
f
\end{array}\right]\right)_{\mathbf{H}} \\
& =\left(\nabla v_{2}, \nabla v_{1}\right)_{\Omega_{\mathrm{s}}}+\left(v_{2}, v_{1}\right)_{\Omega_{\mathrm{s}}}+\left(\Delta v_{1}-v_{1}, v_{2}\right)_{\Omega_{\mathrm{s}}}+(\Delta f-\nabla \pi, f)_{\Omega_{\mathrm{f}}} \\
& =\left(\Delta v_{1}, v_{2}\right)_{\Omega_{\mathrm{s}}}+\left(\nabla v_{2}, \nabla v_{1}\right)_{\Omega_{\mathrm{s}}}+(\Delta f, f)_{\Omega_{\mathrm{f}}}-(\nabla \pi, f)_{\Omega_{\mathrm{f}}}
\end{aligned}
$$

(note that we are implicitly using the fact that $\Delta f, \nabla p \in\left[\left[H^{1}\left(\Omega_{\mathrm{f}}\right)\right]^{n}\right]^{\prime}$ (from (A.5a), and $\Delta v_{1} \in\left[L^{2}\left(\Omega_{\mathrm{s}}\right)\right]^{n}$ (from $\left.(\mathrm{A} .3)\right)$.

(i) A subsequent invocation of Green's Theorem to the first term on the right hand side of (4.1) gives

$$
\left(\Delta v_{1}, v_{2}\right)_{\left[L^{2}\left(\Omega_{\mathrm{s}}\right)\right]^{n}}=-\left(\nabla v_{1}, \nabla v_{2}\right)_{\Omega_{\mathrm{s}}}-\left\langle v_{2}, \frac{\partial v_{1}}{\partial \nu}\right\rangle_{\Gamma_{\mathrm{s}}}
$$


where $\langle\cdot, \cdot\rangle_{\Gamma_{\mathrm{s}}}$ denotes the duality pairing of $\left[H^{1 / 2}\left(\Gamma_{\mathrm{s}}\right)\right]^{n}$ with $\left[H^{-1 / 2}\left(\Gamma_{\mathrm{s}}\right)\right]^{n}$ (we are also implicitly using the fact that the normal vector $\nu(x)$ is interior with respect to $\Omega_{\mathrm{s}}$ ). Using again the definition of $D(\mathcal{A})$, we note that $\left.f\right|_{\Gamma_{\mathrm{s}}}=$ $\left.v_{2}\right|_{\Gamma_{\mathrm{s}}}\left(\right.$ from (A.4)), and $\frac{\partial f}{\partial \nu}-\frac{\partial v_{1}}{\partial \nu}=\pi \nu$ on $\Gamma_{\mathrm{s}}($ from (A.5b)), which combined with (4.2) yields

$$
\left(\nabla v_{1}, \nabla v_{2}\right)_{\Omega_{\mathrm{s}}}+\left(\Delta v_{1}, v_{2}\right)_{\Omega_{\mathrm{s}}}=\left\langle\pi, v_{2} \cdot \nu\right\rangle_{\Gamma_{\mathrm{s}}}-\left\langle f, \frac{\partial f}{\partial \nu}\right\rangle_{\Gamma_{\mathrm{s}}} .
$$

(ii) For the third term on the right hand side of (4.1): Another invocation of Green's Theorem, and the fact that $\left.f\right|_{\Gamma_{\mathrm{f}}}=0$ (from (A.2)) yields

$$
(\Delta f, f)_{\Omega_{\mathrm{f}}}=-\int_{\Omega_{\mathrm{f}}}|\nabla f|^{2} d \Omega_{\mathrm{f}}+\left\langle f, \frac{\partial f}{\partial \nu}\right\rangle_{\Gamma_{\mathrm{s}}} .
$$

(iii) For the fourth term on the right hand side of (4.1): By Green's formula, (A.2), the divergence free imposition in (A.1), and (A.4),

$$
-(\nabla \pi, f)_{\Omega_{\mathrm{f}}}=-\langle\pi, f \cdot \nu\rangle_{\partial \Omega_{\mathrm{f}}}+(\pi, \operatorname{div}(f))_{\Omega_{\mathrm{f}}}=-\left\langle\pi, v_{2} \cdot \nu\right\rangle_{\Gamma_{\mathrm{s}}} .
$$

Applying now (4.3)-(4.5) to the right hand side of (4.1) produces

$$
\left(\mathcal{A}\left[\begin{array}{c}
v_{1} \\
v_{2} \\
f
\end{array}\right],\left[\begin{array}{c}
v_{1} \\
v_{2} \\
f
\end{array}\right]\right)_{\mathbf{H}}=-\int_{\Omega_{\mathrm{f}}}|\nabla f|^{2} d \Omega_{\mathrm{f}} \leq 0,
$$

which demonstrates the asserted dissipativity of $\mathcal{A}$.

4.2. The proof of the maximality of $\mathcal{A}: D(\mathcal{A}) \subset \mathbf{H} \rightarrow \mathbf{H}$. As we have said, our maximality argument here is very different than that presented in [1] in that it is constructive. It will make use of the classical Babuška-Brezzi Theorem, which we recall here:

TheOREM 5 (see, e.g., p. 116 of [11]). Let $\Sigma, \Upsilon$ be Hilbert spaces and $a: \Sigma \times \Sigma \rightarrow \mathbb{R}, b: \Sigma \times \Upsilon \rightarrow \mathbb{R}$ bilinear forms which are continuous. Let

$$
Z=\{\eta \in \Sigma: b(\eta, \varrho)=0 \text { for every } \varrho \in \Upsilon\} .
$$

Assume that $a(\cdot, \cdot)$ is $Z$-elliptic, i.e., there exists a constant $\alpha>0$ such that

$$
a(\eta, \eta) \geq \alpha\|\eta\|_{\Sigma}^{2} \quad \text { for every } \eta \in Z .
$$

Assume further that there exists a constant $\beta>0$ such that

$$
\sup _{\tau \in \Sigma} \frac{b(\tau, \varrho)}{\|\tau\|_{\Sigma}} \geq \beta\left\|_{\varrho}\right\|_{\Upsilon} \quad \text { for every } \varrho \in \Upsilon .
$$

Then for any $\kappa \in \Sigma$ and $l \in \Upsilon$, there exists a unique pair $(\widehat{\eta}, \widehat{\varrho}) \in \Sigma \times \Upsilon$ such that

$$
\begin{aligned}
a(\widehat{\eta}, \tau)+b(\tau, \widehat{\varrho}) & =(\kappa, \tau)_{\Sigma} & & \text { for every } \tau \in \Sigma, \\
b(\widehat{\eta}, \varrho) & =(l, \varrho)_{V} & & \text { for every } \varrho \in \Upsilon .
\end{aligned}
$$


We will use this result to show that for any $\lambda>0$, Range $(\lambda I-\mathcal{A})=\mathbf{H}$. To this end, we consider the task of finding, for given $\left[v_{1}^{*}, v_{2}^{*}, f^{*}\right] \in \mathbf{H}$, a solution $\left[v_{1} v_{2}, f\right] \in D(\mathcal{A})$ of the equation

$$
(\lambda I-\mathcal{A})\left[\begin{array}{c}
v_{1} \\
v_{2} \\
f
\end{array}\right]=\left[\begin{array}{l}
v_{1}^{*} \\
v_{2}^{*} \\
f^{*}
\end{array}\right],
$$

where again $\mathcal{A}$ is as defined in (3.2) and (A.1)-(A.5). Componentwise, the equation yields:

$$
\begin{aligned}
& {\left[v_{1}, v_{2}, f\right] \in\left[H^{1}\left(\Omega_{\mathrm{s}}\right)\right]^{n} \times\left[H^{1}\left(\Omega_{\mathrm{s}}\right)\right]^{n} \times\left(\left[H^{1}\left(\Omega_{\mathrm{f}}\right)\right]^{n} \cap \mathcal{H}_{\mathrm{f}}\right),} \\
& \lambda v_{1}-v_{2}=v_{1}^{*} \quad \text { in }\left[H^{1}\left(\Omega_{\mathrm{s}}\right)\right]^{n}, \\
& \text { (4.10) } \quad \lambda v_{2}-\Delta v_{1}+v_{1}=v_{2}^{*} \quad \text { in }\left[L^{2}\left(\Omega_{\mathrm{S}}\right)\right]^{n} \text {, }
\end{aligned}
$$$$
\text { (4.11) } \quad \lambda f-\Delta f+\nabla \pi=f^{*} \quad \text { in } \mathcal{H}_{\mathrm{f}} \text {. }
$$

As $\left[v_{1}, v_{2}, f\right] \in D(\mathcal{A})$, we also have the additional relations

$$
\begin{array}{rlrl}
\left.f\right|_{\Gamma_{\mathrm{f}}} & =0 & & \text { on } \Gamma_{\mathrm{f}}, \\
\left.f\right|_{\Gamma_{\mathrm{s}}} & =v_{2} & & \text { on } \Gamma_{\mathrm{s}}, \\
\frac{\partial f}{\partial \nu}-\frac{\partial v_{1}}{\partial \nu} & =\pi \nu & & \text { in } \Gamma_{\mathrm{s}}, \\
\operatorname{div}(f)=0 & & \text { a.e. in } \Omega_{\mathrm{f}},
\end{array}
$$

(note that from (A.3) and (A.5), the equality in (4.14) is taken to be in $\left.\left[H^{-1 / 2}\left(\Gamma_{\mathrm{s}}\right)\right]^{n}\right)$. Substituting (4.9) into the PDE (4.10) and the boundary condition (4.13) yields now the BVP

$$
\begin{cases}\lambda^{2} v_{1}-\Delta v_{1}+v_{1}=v_{2}^{*}+\lambda v_{1}^{*} & \text { in } \Omega_{\mathrm{s}}, \\ v_{1}=\frac{1}{\lambda}\left[f+v_{1}^{*}\right] & \text { on } \Gamma_{\mathrm{s}} .\end{cases}
$$

We will write out the "solution" of this system. To do this, we first define $D_{\lambda}:\left[L^{2}\left(\Gamma_{\mathrm{s}}\right)\right]^{n} \rightarrow\left[L^{2}\left(\Omega_{\mathrm{s}}\right)\right]^{n}$ as follows: $g=D_{\lambda}(h)$ if and only if $g$ solves the following elliptic problem:

$$
\begin{cases}\left(\lambda^{2}+I-\Delta\right) g=0 & \text { in } \Omega_{\mathrm{s}}, \\ \left.g\right|_{\Gamma_{\mathrm{s}}}=h & \text { on } \Gamma_{\mathrm{s}} .\end{cases}
$$

By elliptic theory, $D_{\lambda} \in \mathcal{L}\left(\left[H^{r}\left(\Gamma_{\mathrm{s}}\right)\right]^{n},\left[H^{r+1 / 2}\left(\Omega_{\mathrm{S}}\right)\right]^{n}\right)$ for all real $r$ (see [14]). Secondly, we define $\mathbb{A}_{\lambda}: D\left(\mathbb{A}_{\lambda}\right) \rightarrow\left[L^{2}\left(\Omega_{\mathrm{S}}\right)\right]^{n}$ by

$$
\mathbb{A}_{\lambda} \omega=\left(\lambda^{2}+I-\Delta\right) \omega, \quad D\left(\mathbb{A}_{\lambda}\right)=H^{2}\left(\Omega_{\mathrm{s}}\right) \cap H_{0}^{1}\left(\Omega_{\mathrm{s}}\right) .
$$

As defined, $\mathbb{A}_{\lambda}$ is positive self-adjoint, with bounded inverse on $\left[L^{2}\left(\Omega_{\mathrm{S}}\right)\right]^{n}$. With the operators defined in (4.17) and (4.18), the solution $v_{1}$ of (4.16) then admits of the expression

$$
v_{1}=\frac{1}{\lambda} D_{\lambda}\left(\left.f\right|_{\Gamma_{\mathrm{s}}}\right)+\frac{1}{\lambda} D_{\lambda}\left(\left.v_{1}^{*}\right|_{\Gamma_{\mathrm{s}}}\right)+\mathbb{A}_{\lambda}^{-1}\left(v_{2}^{*}+\lambda v_{1}^{*}\right) \in\left[H^{1}\left(\Omega_{\mathrm{s}}\right)\right]^{n} .
$$


Since the variable $f$ is unknown, we of course have not really solved anything yet. But let us turn our attention now to finding the fluid component of (4.7), which solves the system (4.11), vanishes on $\Gamma_{\mathrm{f}}$, and is divergence free. Using the Hilbert space $\mathbf{H}_{\Gamma_{\mathrm{f}}, 0}^{1}\left(\Omega_{\mathrm{f}}\right) \equiv\left\{\phi \in\left[H^{1}\left(\Omega_{\mathrm{f}}\right)\right]^{n}:\left.\phi\right|_{\Gamma_{\mathrm{f}}}=0\right\}$, we multiply (4.11) by $\phi \in \mathbf{H}_{\Gamma_{\mathrm{f}}, 0}^{1}\left(\Omega_{\mathrm{f}}\right)$, and subsequently invoke Green's formula, to obtain

$$
\begin{aligned}
\lambda(f, \phi)_{\Omega_{\mathrm{f}}}+(\nabla f, \nabla \phi)_{\Omega_{\mathrm{f}}}-\langle\phi & \left., \frac{\partial f}{\partial \nu}\right\rangle_{\Gamma_{d}}+(\nabla \pi, \phi)_{\Omega_{\mathrm{f}}} \\
& =\left(f^{*}, \phi\right)_{\Omega_{\mathrm{f}}} \quad \text { for all } \phi \in \mathbf{H}_{\Gamma_{\mathrm{f}}, 0}^{1}\left(\Omega_{\mathrm{f}}\right)
\end{aligned}
$$

(in this computation, we are again implicitly using the fact that $\left[v_{1}, v_{2}, f\right] \in$ $\left.D(\mathcal{A}) \Rightarrow f \in\left[H^{1}\left(\Omega_{\mathrm{f}}\right)\right]^{n}, \Delta f \in\left(\left[H^{1}\left(\Omega_{\mathrm{f}}\right)\right]^{n}\right)^{\prime}, \partial f / \partial \nu \in\left[H^{-1 / 2}(\partial \Omega)\right]^{n}\right)$. Moreover, since $\pi \in L^{2}\left(\Omega_{\mathrm{f}}\right)$ (from (A.1)), and $\nabla \pi \in\left(\left[H^{1}\left(\Omega_{\mathrm{f}}\right)\right]^{n}\right)^{\prime}$ and $\left.\pi\right|_{\Gamma} \in H^{-1 / 2}\left(\partial \Omega_{\mathrm{s}}\right)$ (from (A.5a)), we can use Green's formula to have

$$
(\nabla \pi, \phi)_{\Omega_{\mathrm{f}}}=-(\pi, \operatorname{div}(\phi))_{\Omega_{\mathrm{f}}}+\langle\pi, \phi \cdot \nu\rangle_{\Gamma_{\mathrm{s}}} \quad \text { for all } \phi \in \mathbf{H}_{\Gamma_{\mathrm{f}}, 0}^{1}\left(\Omega_{\mathrm{f}}\right) .
$$

Combining relations (4.20) and (4.21), and considering the boundary condition in (A.5b), we then have the variational relation

$$
\begin{aligned}
\lambda(f, \phi)_{\Omega_{\mathrm{f}}}+(\nabla f, \nabla \phi)_{\Omega_{\mathrm{f}}}-\left\langle\frac{\partial v_{1}}{\partial \nu},\left.\phi\right|_{\Gamma_{\mathrm{s}}}\right\rangle_{\Gamma_{\mathrm{s}}}-(\pi, \operatorname{div}(\phi))_{\Omega_{\mathrm{f}}} \\
\quad=\left(f^{*}, \phi\right)_{\Omega_{\mathrm{f}}} \quad \text { for all } \phi \in \mathbf{H}_{\Gamma_{\mathrm{f}}, 0}^{1}\left(\Omega_{\mathrm{f}}\right) .
\end{aligned}
$$

We further refine the boundary term on the left hand side: With the elliptic map $D_{\lambda}$ as defined in (4.17), and recalling the elliptic BVP (4.16) satisfied by $v_{1}$, we have, for all $\phi \in \mathbf{H}_{\Gamma_{\mathrm{f}}, 0}^{1}\left(\Omega_{\mathrm{f}}\right)$,

$$
\begin{aligned}
& -\left\langle\frac{\partial v_{1}}{\partial \nu},\left.\phi\right|_{\Gamma_{\mathrm{s}}}\right\rangle_{\Gamma_{\mathrm{s}}}=\left(\Delta v_{1}, D_{\lambda}\left(\left.\phi\right|_{\Gamma_{\mathrm{s}}}\right)\right)_{\Omega_{\mathrm{s}}}+\left(\nabla v_{1}, \nabla D_{\lambda}\left(\left.\phi\right|_{\Gamma_{\mathrm{s}}}\right)\right)_{\Omega_{\mathrm{s}}} \\
& =\left(\nabla v_{1}, \nabla D_{\lambda}\left(\left.\phi\right|_{\Gamma_{\mathrm{s}}}\right)\right)_{\Omega_{\mathrm{s}}}+\left(\left[\lambda^{2}+1\right] v_{1}-v_{2}^{*}-\lambda v_{1}^{*}, D_{\lambda}\left(\left.\phi\right|_{\Gamma_{\mathrm{s}}}\right)\right)_{\Omega_{\mathrm{s}}}
\end{aligned}
$$

(note that we are also implicitly using the fact that the normal vector $\nu$ is interior to $\Omega_{\mathrm{s}}$ ). Substituting the expression (4.23) into the right hand side of (4.22), and recalling (4.19), we finally arrive at the following abstract equation in unknowns $f$ and $\pi$ :

$$
a_{\lambda}(f, \phi)-(\pi, \operatorname{div}(\phi))_{\Omega_{\mathrm{f}}}=\mathbf{F}(\phi) \quad \text { for all } \phi \in \mathbf{H}_{\Gamma_{\mathrm{f}}, 0}^{1}\left(\Omega_{\mathrm{f}}\right),
$$

where the bilinear form $a_{\lambda}(\cdot, \cdot): \mathbf{H}_{\Gamma_{\mathrm{f}}, 0}^{1}\left(\Omega_{\mathrm{f}}\right) \times \mathbf{H}_{\Gamma_{\mathrm{f}}, 0}^{1}\left(\Omega_{\mathrm{f}}\right) \rightarrow \mathbb{R}$ is defined by

$$
\begin{gathered}
a_{\lambda}(\psi, \phi)=\lambda(\psi, \phi)_{\Omega_{\mathrm{f}}}+(\nabla \psi, \nabla \phi)_{\Omega_{\mathrm{f}}}+\frac{1}{\lambda}\left(\nabla D_{\lambda}\left(\left.\psi\right|_{\Gamma_{\mathrm{s}}}\right), \nabla D_{\lambda}\left(\left.\phi\right|_{\Gamma_{\mathrm{s}}}\right)\right)_{\Omega_{\mathrm{s}}} \\
+\frac{\lambda^{2}+1}{\lambda}\left(D_{\lambda}\left(\left.\psi\right|_{\Gamma_{\mathrm{s}}}\right), D_{\lambda}\left(\left.\phi\right|_{\Gamma_{\mathrm{s}}}\right)\right)_{\Omega_{\mathrm{s}}} \quad \text { for all } \psi, \phi \in \mathbf{H}_{\Gamma_{\mathrm{f}}, 0}^{1}\left(\Omega_{\mathrm{f}}\right),
\end{gathered}
$$


and the forcing term $\mathbf{F} \in\left[\mathbf{H}_{\Gamma_{\mathrm{f}}, 0}^{1}\left(\Omega_{\mathrm{f}}\right)\right]^{\prime}$ is given by

$$
\mathbf{F}(\phi) \equiv\left(f^{*}, \phi\right)_{\Omega_{\mathrm{f}}}+\left(v_{2}^{*}+\lambda v_{1}^{*}, D_{\lambda}\left(\left.\phi\right|_{\Gamma_{\mathrm{s}}}\right)\right)_{\Omega_{\mathrm{s}}}
$$

$-\left(\lambda^{2}+1\right)\left(\frac{1}{\lambda} D_{\lambda}\left(\left.v_{1}^{*}\right|_{\Gamma_{\mathrm{s}}}\right)+\mathbb{A}_{\lambda}^{-1}\left(v_{2}^{*}+\lambda v_{1}^{*}\right), D_{\lambda}\left(\left.\phi\right|_{\Gamma_{\mathrm{s}}}\right)\right)_{\Omega_{\mathrm{s}}}$ $-\left(\nabla D_{\lambda}\left(\left.\frac{1}{\lambda} v_{1}^{*}\right|_{\Gamma_{\mathrm{s}}}\right)+\nabla \mathbb{A}_{\lambda}^{-1}\left[v_{2}^{*}+\lambda v_{1}^{*}\right], \nabla D_{\lambda}\left(\left.\phi\right|_{\Gamma_{\mathrm{s}}}\right)\right)_{\Omega_{\mathrm{s}}}$ for all $\phi \in \mathbf{H}_{\Gamma_{\mathrm{f}}, 0}^{1}\left(\Omega_{\mathrm{f}}\right)$.

If we now define the bilinear form $b(\cdot, \cdot): \mathbf{H}_{\Gamma_{\mathrm{f}}, 0}^{1}\left(\Omega_{\mathrm{f}}\right) \times L^{2}\left(\Omega_{\mathrm{f}}\right) \rightarrow \mathbb{R}$ by

$$
b(\phi, \varrho) \equiv-(\varrho, \operatorname{div}(\phi))_{L^{2}\left(\Omega_{\mathrm{f}}\right)}
$$

for all $\phi \in \mathbf{H}_{\Gamma_{\mathrm{f}}, 0}^{1}\left(\Omega_{\mathrm{f}}\right)$ and $\varrho \in L^{2}\left(\Omega_{\mathrm{f}}\right)$, then we are steered into the problem of finding a pair $[f, \pi] \in \mathbf{H}_{\Gamma_{\mathrm{f}}, 0}^{1}\left(\Omega_{\mathrm{f}}\right) \times L^{2}\left(\Omega_{\mathrm{f}}\right)$ which solves

$$
\begin{aligned}
a_{\lambda}(f, \phi)+b(\phi, \pi) & =\mathbf{F}(\phi) & & \text { for all } \phi \in \mathbf{H}_{\Gamma_{\mathrm{f}}, 0}^{1}\left(\Omega_{\mathrm{f}}\right), \\
b(f, \varrho) & =0 & & \text { for all } \varrho \in L^{2}\left(\Omega_{\mathrm{f}}\right)
\end{aligned}
$$

(note that the second equation in this system comes from the need to have the fluid component of the solution to (4.7) divergence free).

LEMMA 6. The coupled variational system of equations (4.27), for the given data $\left[v_{1}^{*}, v_{2}^{*}, f^{*}\right] \in \mathbf{H}$, has a unique solution $[f, \pi] \in \mathbf{H}_{\Gamma_{\mathrm{f}}, 0}^{1}\left(\Omega_{\mathrm{f}}\right) \times L^{2}\left(\Omega_{\mathrm{f}}\right)$.

Proof. We wish to employ Theorem 5 , with $\Sigma \equiv \mathbf{H}_{\Gamma_{\mathrm{f}}, 0}^{1}\left(\Omega_{\mathrm{f}}\right), \Upsilon \equiv L^{2}\left(\Omega_{\mathrm{f}}\right)$, and

$$
Z \equiv\left\{\phi \in \mathbf{H}_{\Gamma_{\mathrm{f}}, 0}^{1}\left(\Omega_{\mathrm{f}}\right): \operatorname{div}(\phi)=0\right\}
$$

As defined respectively in (4.24) and $(4.26), a_{\lambda}(\cdot, \cdot)$ and $b(\cdot, \cdot)$ are readily seen to be continuous (after taking in account the regularity of $D_{\lambda}$ and $\mathbb{A}_{\lambda}$ in (4.17) and (4.18).

Moreover, $a_{\lambda}(\cdot, \cdot)$ is elliptic in all of $\mathbf{H}_{\Gamma_{\mathrm{f}}, 0}^{1}\left(\Omega_{\mathrm{f}}\right)$ : In fact, using the definition of $a_{\lambda}(\cdot, \cdot)$ in $(4.24)$, we have, for all $\phi \in \mathbf{H}_{\Gamma_{\mathrm{f}}, 0}^{1}\left(\Omega_{\mathrm{f}}\right)$,

$$
a_{\lambda}(\phi, \phi) \geq\|\nabla \phi\|_{\Omega_{\mathrm{f}}}^{2}
$$

which establishes the asserted ellipticity (of course we are also using Poincaré's inequality, valid here since the test function $\phi$ vanishes on $\Gamma_{\mathrm{f}}$ ). The main issue here, then, is the verification of the inf-sup condition (4.6). To this end, let $\eta \in L^{2}\left(\Omega_{\mathrm{f}}\right)$ be given. Subsequently, let $\omega \in \mathbf{H}_{\Gamma_{\mathrm{f}}, 0}^{1}\left(\Omega_{\mathrm{f}}\right)$ be the solution of the boundary value problem

$$
\begin{cases}\operatorname{div}(\omega)=-\eta & \text { in } \Omega_{\mathrm{f}}, \\ \left.\omega\right|_{\Gamma_{\mathrm{f}}}=0 & \text { on } \Gamma_{\mathrm{f}}, \\ \left.\omega\right|_{\Gamma_{\mathrm{s}}}=-\frac{\left(\int \eta d \Omega_{\mathrm{s}}\right)}{\operatorname{meas}\left(\Gamma_{\mathrm{s}}\right)} \nu & \text { on } \Gamma_{\mathrm{s}} .\end{cases}
$$


That such a solution $\omega \in \mathbf{H}_{\Gamma_{\mathrm{f}}, 0}^{1}\left(\Omega_{\mathrm{f}}\right)$ exists follows from [9, p. 127] (inasmuch as the data in (4.28) satisfy the needed compatibility conditions). Moreover, one has in [9] the following estimate for the solution for some positive constant $C$ :

$$
\|\nabla \omega\|_{\Omega_{\mathrm{f}}} \leq C\|\eta\|_{\Omega_{\mathrm{f}}} .
$$

We then have, for the given $L^{2}$-function $\eta$,

$$
\begin{aligned}
\sup _{\phi \in \mathbf{H}_{\Gamma_{\mathrm{f}}, 0}^{1}\left(\Omega_{\mathrm{f}}\right)} \frac{b(\phi, \eta)}{\|\nabla \phi\|_{\Omega_{\mathrm{f}}}} & =\sup _{\phi \in \mathbf{H}_{\Gamma_{\mathrm{f}}, 0}^{1}\left(\Omega_{\mathrm{f}}\right)} \frac{-\int \eta \operatorname{div}(\phi) d \Omega_{\mathrm{f}}}{\|\nabla \phi\|_{\Omega_{\mathrm{f}}}} \\
& \geq \frac{-\int \eta \operatorname{div}(\omega) d \Omega_{\mathrm{f}}}{\|\nabla \omega\|_{\Omega_{\mathrm{f}}}}=\frac{\int \eta^{2} d \Omega_{\mathrm{f}}}{\|\nabla \omega\|_{\Omega_{\mathrm{f}}}}=\frac{\|\eta\|_{\Omega_{\mathrm{f}}}^{2}}{\|\nabla \omega\|_{\Omega_{\mathrm{f}}}} \geq \frac{1}{C}\|\eta\|_{\Omega_{\mathrm{f}}} .
\end{aligned}
$$

Thus, the inf-sup condition (4.6) is satisfied, and the Babuška-Brezzi Theorem can be applied so as to yield a unique pair $[f, \pi] \in \mathbf{H}_{\Gamma_{\mathrm{f}}, 0}^{1}\left(\Omega_{\mathrm{f}}\right) \times L^{2}\left(\Omega_{\mathrm{f}}\right)$ which solves the system (4.27).

We now finish the proof of maximality by using the pair $[f, \pi]$ obtained from Lemma 6 , and the relation (4.19), to recover the solution components $v_{1}$ and $v_{2}$ of $(4.7)$ (and of course we must also justify that $\left[v_{1}, v_{2}, f\right] \in D(\mathcal{A})$, as given in (A.1)-(A.5)).

To start, since $[f, \pi] \in \mathbf{H}_{\Gamma_{\mathrm{f}}, 0}^{1}\left(\Omega_{\mathrm{f}}\right) \times L^{2}\left(\Omega_{\mathrm{f}}\right)$ solves the variational relation (4.27), we infer from the second equation that

$$
f \in \mathbf{H}_{\Gamma_{\mathrm{f}}, 0}^{1}\left(\Omega_{\mathrm{f}}\right) \quad \text { with } \operatorname{div}(f)=0 \text { in } \Omega_{\mathrm{f}} .
$$

Moreover, from (4.27) we infer, after integrating by parts in the first equation, that $f \in \mathbf{H}_{\Gamma_{\mathrm{f}}, 0}^{1}\left(\Omega_{\mathrm{f}}\right) \cap \mathcal{H}_{\mathrm{f}}$ satisfies

$$
\lambda(f, \phi)_{\Omega_{\mathrm{f}}}-(\Delta f, \phi)_{\Omega_{\mathrm{f}}}+\left(\nabla \pi_{0}, \phi\right)_{\Omega_{\mathrm{f}}}=\left(f^{*}, \phi\right)_{\Omega_{\mathrm{f}}} \quad \text { for all } \phi \in\left[D\left(\Omega_{\mathrm{f}}\right)\right]^{n} .
$$

Thus,

$$
\lambda f-\Delta f+\nabla \pi_{0}=f^{*} .
$$

Hence, from Lemma 2, we have the additional regularity for the pair $[f, \pi] \in$ $\left(\mathbf{H}_{\Gamma_{\mathrm{f}}, 0}^{1}\left(\Omega_{\mathrm{f}}\right) \cap \mathcal{H}_{\mathrm{f}}\right) \times L^{2}\left(\Omega_{\mathrm{f}}\right):$

$$
\begin{array}{cr}
\left.\pi_{0}\right|_{\partial \Omega_{\mathrm{f}}} \in H^{-1 / 2}\left(\partial \Omega_{\mathrm{f}}\right), & \left.\frac{\partial \pi_{0}}{\partial \nu}\right|_{\partial \Omega_{\mathrm{f}}} \in H^{-3 / 2}\left(\partial \Omega_{\mathrm{f}}\right), \\
\left.\frac{\partial f}{\partial \nu}\right|_{\partial \Omega_{\mathrm{f}}} \in\left[H^{-1 / 2}\left(\partial \Omega_{\mathrm{f}}\right)\right]^{n}, & {[(\Delta f) \cdot \nu]_{\partial \Omega_{\mathrm{f}}} \in H^{-3 / 2}\left(\partial \Omega_{\mathrm{f}}\right) .}
\end{array}
$$

In turn, we can recover the elastic variable $v_{1}$ by means of the relation in (4.19). Thus, by construction we have

$$
v_{1} \in\left[H^{1}\left(\Omega_{\mathrm{s}}\right)\right]^{n} \quad \begin{cases}\lambda^{2} v_{1}-\Delta v_{1}+v_{1}=v_{2}^{*}+\lambda v_{1}^{*} & \text { in } \Omega_{\mathrm{s}}, \\ v_{1}=\frac{1}{\lambda}\left[f+v_{1}^{*}\right] & \text { on } \Gamma_{\mathrm{s}} .\end{cases}
$$


From this expression, we also infer that

$$
\Delta v_{1} \in\left[L^{2}\left(\Omega_{\mathrm{S}}\right)\right]^{n} .
$$

We now show that this newly found variable $v_{1}$ also satisfies the needed boundary relation (4.14). Since $[f, \pi] \in \mathbf{H}_{\Gamma_{\mathrm{f}}, 0}^{1}\left(\Omega_{\mathrm{f}}\right) \times L^{2}\left(\Omega_{\mathrm{f}}\right)$ solves (4.27), we then have the relation (after again using the expression for $v_{1}$ in (4.19))

$$
\begin{aligned}
\lambda(f, \phi)_{\Omega_{\mathrm{f}}} & +(\nabla f, \nabla \phi)_{\Omega_{\mathrm{f}}}+\left(\nabla v_{1}, \nabla D_{\lambda}\left(\left.\phi\right|_{\Gamma_{\mathrm{s}}}\right)\right)_{\Omega_{\mathrm{s}}} \\
& +\left[\lambda^{2}+1\right]\left(v_{1}, D_{\lambda}\left(\left.\phi\right|_{\Gamma_{\mathrm{s}}}\right)\right)_{\Omega_{\mathrm{s}}}-(\pi, \operatorname{div}(\phi))_{\Omega_{\mathrm{f}}} \\
= & \left(f^{*}, \phi\right)_{\Omega_{\mathrm{f}}}+\left(v_{2}^{*}+\lambda v_{1}^{*}, D_{\lambda}\left(\left.\phi\right|_{\Gamma_{\mathrm{s}}}\right)\right)_{\Omega_{\mathrm{s}}} \quad \text { for all } \phi \in \mathbf{H}_{\Gamma_{\mathrm{f}}, 0}^{1}\left(\Omega_{\mathrm{f}}\right) .
\end{aligned}
$$

Integrating by parts, and using the trace regularity posted in (4.31), we have

$$
\begin{aligned}
\lambda(f, \phi)_{\Omega_{\mathrm{f}}}-(\Delta f, \phi)_{\Omega_{\mathrm{f}}}+\left\langle\frac{\partial f}{\partial \nu},\left.\phi\right|_{\Gamma_{\mathrm{s}}}\right\rangle_{\Gamma_{\mathrm{s}}}-\left(\Delta v_{1}, D_{\lambda}\left(\left.\phi\right|_{\Gamma_{\mathrm{s}}}\right)\right)_{\Omega_{\mathrm{s}}}-\left\langle\frac{\partial v_{1}}{\partial \nu},\left.\phi\right|_{\Gamma_{\mathrm{s}}}\right\rangle_{\Gamma_{\mathrm{s}}} \\
+\left[\lambda^{2}+1\right]\left(v_{1}, D_{\lambda}\left(\left.\phi\right|_{\Gamma_{\mathrm{s}}}\right)\right)_{\Omega_{\mathrm{s}}}+(\nabla \pi, \phi)_{\Omega_{\mathrm{f}}}-\left\langle\pi \nu,\left.\phi\right|_{\Gamma_{\mathrm{s}}}\right\rangle_{\Gamma_{\mathrm{s}}} \\
=\left(f^{*}, \phi\right)_{\Omega_{\mathrm{f}}}+\left(v_{2}^{*}+\lambda v_{1}^{*}, D_{\lambda}\left(\left.\phi\right|_{\Gamma_{\mathrm{s}}}\right)\right)_{\Omega_{\mathrm{s}}} \quad \text { for all } \phi \in \mathbf{H}_{\Gamma_{\mathrm{f}}, 0}^{1}\left(\Omega_{\mathrm{f}}\right) .
\end{aligned}
$$

Applying now the equations in (4.30) and (4.32), solved by $f$ and $v_{1}$ respectively, we obtain

$$
\left\langle\frac{\partial f}{\partial \nu},\left.\phi\right|_{\Gamma_{\mathrm{s}}}\right\rangle_{\Gamma_{\mathrm{s}}}-\left\langle\frac{\partial v_{1}}{\partial \nu},\left.\phi\right|_{\Gamma_{\mathrm{s}}}\right\rangle_{\Gamma_{\mathrm{s}}}-\left\langle\pi \nu,\left.\phi\right|_{\Gamma_{\mathrm{s}}}\right\rangle_{\Gamma_{\mathrm{s}}}=0 \quad \text { for all } \phi \in \mathbf{H}_{\Gamma_{\mathrm{f}}, 0}^{1}\left(\Omega_{\mathrm{f}}\right),
$$

from which (and the surjectivity of the Sobolev trace map) we now infer that

$$
\left[\frac{\partial f}{\partial \nu}-\frac{\partial v_{1}}{\partial \nu}\right]_{\Gamma_{\mathrm{s}}}=\left.\pi \nu\right|_{\Gamma_{\mathrm{s}}} \quad \text { in }\left[H^{-1 / 2}\left(\Gamma_{\mathrm{s}}\right)\right]^{n}
$$

Finally, we set

$$
v_{2}=\lambda v_{1}-v_{1}^{*} \in\left[H^{1}\left(\Omega_{\mathrm{s}}\right)\right]^{n} .
$$

From the boundary value in (4.16), we see in turn that

$$
\left.v_{2}\right|_{\Gamma_{\mathrm{s}}}=\left[f+v_{1}^{*}\right]_{\Gamma_{\mathrm{s}}}-\left.v_{1}^{*}\right|_{\Gamma_{\mathrm{s}}}=\left.f\right|_{\Gamma_{\mathrm{s}}} .
$$

Collecting now the properties (4.29)-(4.33) and (4.34)-(4.36) establishes the range condition (4.7), with solution $\left[v_{1}, v_{2}, f\right] \in D(\mathcal{A})$, as prescribed in (A.1)-(A.5). This concludes the proof of maximality.

Having thus shown that the fluid-structure generator $\mathcal{A}: D(\mathcal{A}) \subset \mathbf{H} \rightarrow \mathbf{H}$ is maximal dissipative, contraction semigroup generation follows from the Lumer-Phillips Theorem. The proof of the extra regularity (3.8) follows from a standard energy method applied to the system (1.1)-(1.3), which starts by multiplying the fluid equation of (1.1) by $u$, the elastic equation in (1.1) by $w_{t}$ and integrating in time and space. This concludes the proof of Theorem 3 . 
5. A numerical algorithm for approximating the solution of the fluid-structure equation (4.7). Here, we give a brief description of how the maximality argument set down in Section 4.2 can be used to numerically approximate the solution to the given fluid-structure interactive system. We put emphasis here on formulation and implementation; convergence analysis of the FEM below will be provided in another paper. Also, we will focus on the static equation in (4.7); however, this static equation can in turn be invoked to approximate solutions of the time evolving system (1.1)-(1.3), via the exponential formula

$$
\left[\begin{array}{l}
w(t) \\
w_{t}(t) \\
u(t)
\end{array}\right]=e^{\mathcal{A} t}\left[\begin{array}{l}
w_{0} \\
w_{1} \\
u_{0}
\end{array}\right]=\lim _{n \rightarrow \infty}\left(I-\frac{t}{n} \mathcal{A}\right)^{-n}\left[\begin{array}{l}
w_{0} \\
w_{1} \\
u_{0}
\end{array}\right] \quad \text { for }\left[\begin{array}{l}
w_{0} \\
w_{1} \\
u_{0}
\end{array}\right] \in \mathbf{H}
$$

(see, e.g., p. 91 of [15], as well as the approximation schemes of Corollary 5.6 therein).

5.1. The numerical procedure. We will operate here with a view to finding a finite-dimensional approximation $\left[\left(v_{1}\right)_{h},\left(v_{2}\right)_{h}, f_{h}\right]$ to the solution $\left[v_{1}, v_{2}, f\right]$ of the equation (4.7), for given $\left[v_{1}^{*}, v_{2}^{*}, f^{*}\right]$ of $\mathbf{H}$. With $h$ being (as usual) a parameter of discretization with respect to the geometry $\left\{\Omega_{\mathrm{f}}, \Omega_{\mathrm{s}}\right\}$, then: (i) Let $V_{h} \subset \mathbf{H}_{\Gamma_{\mathrm{f}}, 0}^{1}\left(\Omega_{\mathrm{f}}\right)$ be a finite-dimensional approximating subspace of $\mathbf{H}_{\Gamma_{\mathrm{f}}, 0}^{1}\left(\Omega_{\mathrm{f}}\right)$; (ii) let $\Pi_{h} \subset L^{2}\left(\Omega_{\mathrm{f}}\right)$ be an approximating subspace of $L^{2}\left(\Omega_{\mathrm{f}}\right)$; (iii) let $W_{h} \subset\left[H_{0}^{1}\left(\Omega_{\mathrm{s}}\right)\right]^{n}$ be an approximating subspace of $\left[H_{0}^{1}\left(\Omega_{\mathrm{s}}\right)\right]^{n}$. Our Ritz-Galerkin method will take its cue from the maximality argument outlined in Section 4.2. Namely, we find a pair $\left(f_{h}, \pi_{h}\right)$ which uniquely solves the following variational relation (cf. (4.27)):

$$
\begin{aligned}
a_{\lambda}\left(f_{h}, \phi_{h}\right)+b\left(\phi_{h}, \pi_{h}\right) & =\mathbf{F}\left(\phi_{h}\right) & & \text { for all } \phi_{h} \in V_{h}, \\
b\left(f_{h}, \varrho_{h}\right) & =0 & & \text { for all } \varrho_{h} \in \Pi_{h},
\end{aligned}
$$

where the bilinear forms $a_{\lambda}(\cdot, \cdot)$ and $b(\cdot, \cdot)$ are respectively defined in (4.24) and (4.26), and the functional $\mathbf{F}(\cdot)$ is as given in (4.25) (note that we are making no assumptions that the approximating fluid subspace $V_{h}$ comprises divergence free functions; this is the positive virtue of such a mixed formulation; see [5]). Assuming we can solve the finite-dimensional system for the unknowns $\left[f_{h}, \pi_{h}\right] \in V_{h} \times \Pi_{h}$ (in particular, assuming the approximating subspaces $\left\{V_{h}, \Pi_{h}\right\}$ are such that the inf-sup condition (4.6) is satisfied, see [5]) we can subsequently recover the approximation $\left(v_{1}\right)_{h}$ in the following manner: Let a fixed $v^{*} \in\left[H^{1}\left(\Omega_{\mathrm{s}}\right)\right]^{n}$ satisfy $\left.v^{*}\right|_{\Gamma_{\mathrm{s}}}=\lambda^{-1}\left[f+v_{1}^{*}\right]$. Then with $\widetilde{W}_{h} \equiv\left\{\xi_{h}=\psi_{h}+v^{*}: \psi_{h} \in W_{h}\right\}$, we obtain $\left(v_{1}\right)_{h}$ to be the unique element in $\widetilde{W}_{h}$ which solves

$$
\lambda^{2}\left(\left(v_{1}\right)_{h}, \psi_{h}\right)_{\Omega_{\mathrm{s}}}+\left(\nabla\left[\left(v_{1}\right)_{h}\right], \nabla \psi_{h}\right)_{\Omega_{\mathrm{s}}}=\left(v_{2}^{*}+\lambda v_{1}^{*}, \psi_{h}\right)_{\Omega_{\mathrm{s}}} \text { for all } \psi_{h} \in W_{h}
$$


(cf. (4.16)). In turn, an approximation $\left(v_{2}\right)_{h}$ to the structural term $v_{2}$ can be obtained via the relation

$$
\left(v_{2}\right)_{h}=\lambda\left(v_{1}\right)_{h}-v_{1}^{*}
$$

(cf. (4.9)).

5.2. A numerical example. The procedure described above is considered in the context of a specific numerical example, with solid domain $\Omega_{\mathrm{s}}=$ $(1 / 3,2 / 3)^{2}$, and fluid domain $\Omega_{\mathrm{f}}=(0,1)^{2} \backslash[1 / 3,2 / 3]^{2}$; see Figure 2 .

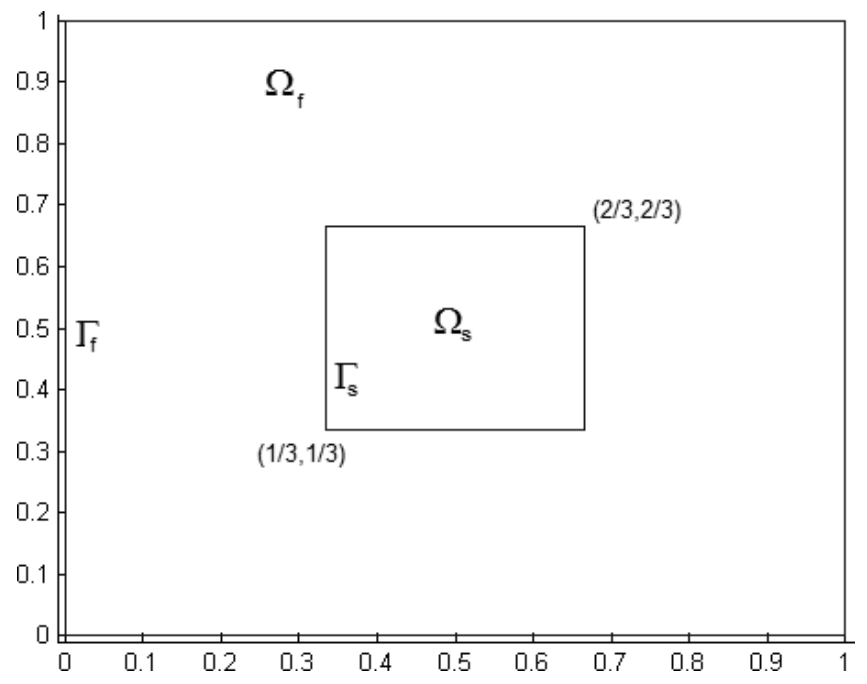

Fig. 2. The fluid-structure geometry of the numerical example

We will consider the task of approximating the solution of equation (4.7), with data

$$
\left[\begin{array}{c}
v_{1}^{*} \\
v_{2}^{*} \\
f^{*}
\end{array}\right]=\lambda\left[\begin{array}{l}
\eta \\
0 \\
0
\end{array}\right],
$$

where $\eta$ is the unique solution of the elliptic problem

$$
\Delta \eta-\eta=0 \quad \text { in } \Omega_{\mathrm{s}}, \quad \frac{\partial \eta}{\partial \nu}=\nu \quad \text { on } \Gamma_{\mathrm{s}}
$$

(where again $\nu$ is the unit normal vector, interior with respect to $\Omega_{\mathrm{S}}$ ). It is shown in [1] that $[\eta, 0,0]$ is an eigenfunction corresponding to the eigenvalue zero of the fluid-structure generator $\mathcal{A}: D(\mathcal{A}) \subset \mathbf{H} \rightarrow \mathbf{H}$, as given in (3.2) and (A.1)-(A.5). (So the actual solution is $\left[v_{1}, v_{2}, f\right]=[\eta, 0,0]$.) For the canonical geometry $\Omega_{\mathrm{S}}=(1 / 3,2 / 3) \times(1 / 3,2 / 3)$, the solution $\eta$ of (5.3) can 
be explicitly computed as:

$\eta(x, y)=$

$\left[\begin{array}{l}\frac{\left[1-\frac{(\cosh (1 / 3)-\cosh (2 / 3)) \sinh (1 / 3)}{\sinh (2 / 3) \cosh (1 / 3)-\sinh (1 / 3) \cosh (2 / 3)}\right] \sinh (x)}{\cosh (1 / 3)}+\frac{(\cosh (1 / 3)-\cosh (2 / 3)) \cosh (x)}{\sinh (2 / 3) \cosh (1 / 3)-\sinh (1 / 3) \cosh (2 / 3)} \\ \frac{\left[1-\frac{(\cosh (1 / 3)-\cosh (2 / 3) \sinh (1 / 3)}{\sinh (2 / 3) \cosh (1 / 3)-\sinh (1 / 3) \cosh (2 / 3)}\right] \sinh (y)}{\cosh (1 / 3)}+\frac{(\cosh (1 / 3)-\cosh (2 / 3)) \cosh (y)}{\sinh (2 / 3) \cosh (1 / 3)-\sinh (1 / 3) \cosh (2 / 3)}\end{array}\right]$.

Moreover, from (A.5b), we see immediately that the pressure function corresponding to data $[\eta, 0,0]$ is $\pi=-1$. This initial data (5.2) thus provides a verifiable test problem for the Ritz-Galerkin method we described above.

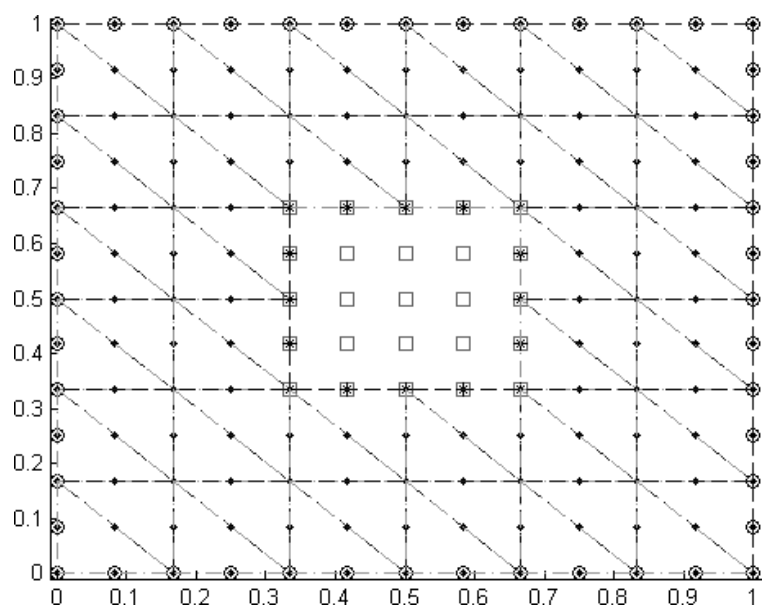

Fig. 3. The fluid finite element mesh

Subsequently, we invoked here a basic FEM for the elliptic BVP (see, e.g. [3]), which involves taking the aforesaid spaces $V_{h}$ and $W_{h}$ to be the linear span of piecewise quadratic basis functions defined on triangular elements, and $\Pi_{h}$ the linear span of piecewise linear basis functions defined over the same triangular elements. In fact, our particular elements are uniform right triangles, but this is only for computational ease here and of course is not necessary in practice; see Figure 3.

Table 1. Finite element solution error

\begin{tabular}{ccccc}
\hline No. of elements & Length of hypothenuse & $\left\|f_{h}-f\right\|_{1, \Omega_{\mathrm{f}}}$ & $\left\|\pi_{h}-\pi\right\|_{L^{2}\left(\Omega_{\mathrm{f}}\right)}$ & $\left\|\left(v_{1}\right)_{h}-v_{1}\right\|_{1, \Omega_{\mathrm{s}}}$ \\
\hline 18 & 0.471405 & $3.36 \cdot 10^{-4}$ & $2.08 \cdot 10^{-4}$ & $3.01 \cdot 10^{-3}$ \\
72 & 0.235702 & $6.12 \cdot 10^{-5}$ & $1.41 \cdot 10^{-5}$ & $7.47 \cdot 10^{-4}$ \\
288 & 0.117851 & $1.11 \cdot 10^{-5}$ & $1.53 \cdot 10^{-6}$ & $1.85 \cdot 10^{-4}$ \\
1152 & 0.058926 & $2.04 \cdot 10^{-6}$ & $1.81 \cdot 10^{-7}$ & $4.58 \cdot 10^{-5}$ \\
4608 & 0.029463 & $3.85 \cdot 10^{-7}$ & $2.39 \cdot 10^{-8}$ & $1.14 \cdot 10^{-5}$ \\
\hline
\end{tabular}


Table 1 details the error between the approximate and actual solution, with $\lambda=1$. In particular, the graphs of the solid displacement approximation $\left(v_{1}\right)_{h}$ are visually identical to those of the true solution $v_{1}=\eta$, with $\eta$ as given above. As we have said, a rigorous convergence analysis will be in our forthcoming paper, and it will include the all-important verification of the discrete inf-sup condition for the mixed variational formulation (5.1); see [5] and references therein.

\section{References}

[1] G. Avalos and R. Triggiani, The coupled PDE system arising in fluid/structure interaction, Part I: explicit semigroup generator and its spectral properties, in: Fluids and Waves, Contemp. Math. 440, Amer. Math. Soc., Providence, RI, 2007, 15-54.

[2] - - - Wellposedness and stability analysis of a coupled Stokes-Lamé system, as a PDE model of certain fluid-structure interactions, preprint, 2007.

[3] O. Axelsson and V. A. Barker, Finite Element Solution of Boundary Value Problems: Theory and Computation, Academic Press, New York, 1984.

[4] V. Barbu, Z. Grujić, I. Lasiecka and A. Tuffaha, Weak and strong solutions in nonlinear fluid-structure interactions, in: Fluids and Waves, Contemp. Math. 440, Amer. Math. Soc., Providence, RI, 2007, 55-82.

[5] F. Brezzi and M. Fortin, Mixed and Hybrid Finite Element Methods, Springer Ser. Comput. Math., Springer, New York, 1991.

[6] P. Constantin and C. Foias, Navier-Stokes Equations, Univ. of Chicago Press, Chicago, 1989.

[7] C. R. Doering and J. D. Gibbon, Applied Analysis of the Navier-Stokes Equations, Cambridge Texts in Appl. Math., Cambridge Univ. Press, New York, 1995.

[8] Q. Du, M. D. Gunzburger, L. S. Hou and J. Lee, Analysis of a linear fluid-structure interaction problem, Discrete Contin. Dynam. Systems 9 (2003), 633-650.

[9] G. P. Galdi, An Introduction to the Mathematical Theory of the Navier-Stokes Equations, Springer Tracts in Natural Philosophy 38, Springer, New York, 1994.

[10] B. Kellogg, Properties of solutions of elliptic boundary value problems, in: The Mathematical Foundations of the Finite Element Method with Applications to Partial Differential Equations, A. K. Aziz (ed.), Academic Press, New York, 1972, 47-81.

[11] S. Kesavan, Topics in Functional Analysis and Applications, Wiley, New York, 1989.

[12] I. Lasiecka and R. Triggiani, Control Theory for Partial Differential Equations, Continuous and Approximation Theories, Vols. I and II, Cambridge Univ. Press, New York, 2000.

[13] J.-L. Lions, Quelques méthodes de résolution des problèmes aux limites non linéaires, Dunod, Paris, 1969.

[14] J.-L. Lions and E. Magenes, Non-Homogeneous Boundary Value Problems and Applications, Vol. I, Springer, 1972.

[15] A. Pazy, Semigroups of Linear Operators and Applications to Partial Differential Equations, Springer, New York, 1983. 
[16] A. E. Taylor and D. C. Lay, Introduction to Functional Analysis, 2nd ed., Wiley, New York, 1980.

Department of Mathematics University of Nebraska-Lincoln Lincoln, NE 68588, U.S.A.

E-mail: gavalos@math.unl.edu

Received on 2.1.2008;

revised version on 20.5.2008 